TITLE:

\title{
A statistical deterioration forecasting method using hidden Markov model for infrastructure management
}

\section{AUTHOR(S):}

Kobayashi, Kiyoshi; Kaito, Kiyoyuki; Lethanh, Nam

\section{CITATION:}

Kobayashi, Kiyoshi ...[et al]. A statistical deterioration forecasting method using hidden Markov model for infrastructure management. Transportation Research Part B:

Methodological 2012, 46(4): 544-561

ISSUE DATE:

2012-03

URL:

http://hdl.handle.net/2433/155096

\section{RIGHT:}

(c) 2011 Elsevier Ltd.; この論文は出版社版でありません。引用の際には 出版社版をご確認ご利用ください。; This is not the published version. Please cite only the published version. 


\title{
A Statistical Deterioration Forecasting Method using Hidden Markov Model for Infrastructure Management
}

\author{
Kiyoshi Kobayashi ${ }^{\mathrm{a}}$, Kiyoyuki Kaito ${ }^{\mathrm{b}}$, Nam Lethanh' ${ }^{\mathrm{c}} \mathrm{a}^{*}$ \\ ${ }^{a}$ Dept. of Urban Management, Graduate School of Engineering, Kyoto Univ., Kyoto 615-8540, Japan \\ ${ }^{b}$ Dept. of Civil Engineering, Graduate School of Engineering,Osaka Univ., Suita, Osaka 565-0871, Japan \\ ${ }^{c}$ Institute of Construction and Infrastructure Management, Swiss Federal Institute of Technology (ETH), Zurich 8093, Switzerland
}

\begin{abstract}
The application of Markov models as deterioration-forecasting tools has been widely documented in the practice of infrastructure management. The Markov chain models employ monitoring data from visual inspection activities over a period of time in order to predict the deterioration progress of infrastructure systems. Monitoring data play a vital part in the managerial framework of infrastructure management. As a matter of course, the accuracy of deterioration prediction and life cycle cost analysis largely depends on the soundness of monitoring data. However, in reality, monitoring data often contain measurement errors and selection biases, which tend to weaken the correctness of estimation results. In this paper, the authors present a hidden Markov model to tackle selection biases in monitoring data. Selection biases are assumed as random variables. Bayesian estimation and Markov Chain Monte Carlo simulation are employed as techniques in tackling the posterior probability distribution, the random generation of condition states, and the model's parameters. An empirical application to the Japanese national road system is presented to demonstrate the applicability of the model. Estimation results highlight the fact that the properties of the Markov transition matrix have greatly improved in comparison with the properties obtained from applying the conventional multi-stage exponential Markov model.
\end{abstract}

"Keywords: Infrastructure management, Hidden Markov model; Measurement errors; Selection bias; Bayesian estimation; MCMC"

\section{Introduction}

Statistical deterioration forecasting with Markov models has been widely documented as an important methodology for hazard analysis in the practice of infrastructure management (Madanat et al,. 1995, Shin and Madanat, 2003; Shahin, 2005). A good example of its application is the PONTIS program (Golabi and Shepard, 1997), which was developed for bridge management systems (BMS). In Markov models, the deterioration of an infrastructure system is represented by the transition probability among its discrete condition states, which reflect the status of its health.

In order to apply Markov models for prediction, it is necessary to employ monitoring data from historical inspections, the quality of which is a decisive factor in the accuracy of estimation results. In infrastructure management practice, however, monitoring data are often marred by errors and bias. Measurement errors can arise from the measurement system itself, the inspector (human or machine), the inspected objects, or problems with data processing and interpretation (Humplick, 1992). Such errors tend to cause bias in the estimation results of deterioration models, especially when there is a small pool of monitoring data.

Researchers have proposed methods of tackling problems related to measurement errors in monitoring data. Efforts have been made to perfect evaluation techniques for quantifying the error term (Cochoran and Cox,. 1968; Grubbs, 1973; Humplick, 1992). In addition, to cope with small sampling populations of monitoring data and

\footnotetext{
* Corresponding author. Tel.: +41-762-072-105; fax: +41-446-331-088.

E-mail address: kkoba@psa.mbox.media.kyoto-u.ac.jp (K.Kobayashi), kaito@ga.eng.osaka-u.ac.jp (K.Kaito), lethanh@ibi.baug.ethz.ch (N. Lethanh)
}

Preprint submitted to Transportation Research: Part B

September, 26th, 2011 
measurement errors, researchers have proposed estimation methodologies using the Bayesian estimation technique (Hong and Prozzi, 2006; Ben-Akiva et al, 1993; Ben-Akiva and Ramaswamy, 1993). To overcome problems in spatial sampling, which is viewed as contributing to measurement errors, Mishalani and Gong (2009) propose an optimization model using the latent Markov decision process (LMDP) to select the best sample size. To our knowledge, a paper by Humplick (1992) is considered the fundamental measurement errors model for later models using LMDP in infrastructure management.

\section{\{Fig. 1 is about here\}}

In the paper of Humplick (1992), the author proposed a factor analytical model focused on differentiating the causes of measurement errors as influenced by three categories: monitoring technologies, specific items, and measurement locations. Measurement errors are quantified by weighting factors for error terms with respect to the three categories. The best technology offering least measurement error becomes preferable in adjusting the error term and defining performance distress. Later research on LMDP then utilizes the estimation results of this measurement error model as input.

Another research orientation toward elimination of measurement errors is with the application of Kalman filter. This mathematical method originated from the field of statistic and has been widely applied in many engineering fields. Theoretically, Kalman filter is an algorithm to generate estimates of the true values of observations and their associated calculated values by predicting a value, estimating the uncertainty of the predicted one, and finally computing a weighted average of the predicted value and the measured value. The weighted average is calculated from the covariance, a measure of the estimated uncertainty of the prediction of the system's state. As the weighted average has a better estimated uncertainty than either alone, the estimates produced by the algorithm tend to be closer to the true values than the original observations (Kalman, 1960). To the best of the authors' knowledge, application of Kalman filters for management of infrastructure system has been recently documented in the papers of Durago-Cohen (2007), Chu and Durago-Cohen (2007), and Chu and Durago-Cohen (2008). Of the cited papers, continuous state-space models are discussed. The performance or condition of an infrastructure system is reflected in a continuous performance indicator. Using the framework of Kalman filter algorithm, authors of the cited papers have successfully discussed and proposed an approach for deterioration prediction within a dynamic infrastructure management system, in which, heterogeneity factors, time-series and panel data are used; effects of interventions over the life cycle of infrastructure system, and measurement errors are included.

The hidden Markov model is a special case of Markov chain model, which has been widely used in several research areas such as image processing, speech recognition, and applied statistics (Robert et al, 2000; MacDonald and Zucchini, 1997; Lawrence, 1989). One of the great advantages of the hidden Markov model is that it allows the unobserved condition state to be captured, eliminating the noise and bias associated with monitoring data. Of cited studies on hidden Markov models, the main focus has been on the accumulation of discrete-value in time series. In addition, several profound research studies on hidden Markov models with unobserved states of regimes can be found in economics and finance engineering literature (Hamilton, 1989; Diebold and Inoue, 2001; Hamilton and Raul, 1994), where the authors attempt to simulate and evaluate the business cycle and switching of regimes by using non-stationary time series. One important finding is that the change of longitudinal data can be simulated by means of transition probability. In addition, research has shown that the transition probability can be identified in the non-linear auto-regressive approach using the Markov chain theory (Hamilton, 1989; Kim and Nelson, 1999). However, a majority of past research on hidden Markov models has proposed estimation methods to uncover unobserved condition states based on true condition states, which must be available as monitoring data. Under such an assumption, it is certain that the true condition states of a system are no longer random variables. This fact is considered as a limitation of the cited research.

Apparently, research on the hidden Markov model has not been applied elsewhere in the literature of infrastructure management. In this paper, we develop a hidden Markov model to tackle a type of measurement errors in monitoring data so that estimation outcomes can be more reliable for practical use. It is noted that the term "measurement errors" in our model is referred as selection bias, which is explained in detail in section 2. In the model, we assume that "true condition states" and "observed condition states" of road sections are random variables. This assumption reflects the real possibility of having selection biases in the system. In another words, we consider selection biases as random variables. This assumption is one of the key features of our model, which is different from the assumption in past models. To describe selection biases as random variables, a mixture of mathematical forms between "true condition states" of road sections and "observed conditions states" or "selection biases" is 
proposed using the conditional probability distribution function. Also in our model, an innovative numerical estimation approach using Bayesian estimation and MCMC simulation is presented to overcome the difficulty of the complete likelihood function so that optimal values of the model's parameters can be obtained.

In comparison with the proposed methods in the research work of Humplick (1992) and the use of Kalman filter in the papers of Durago-Cohen (2007), Chu and Durago-Cohen (2007), and Chu and Durago-Cohen (2008), our paper is different from them in two fundamental research standpoints. Firstly, in our paper, we basically deal with a special type of measurement errors called selection bias. Precisely, we assume that measurements carried out by monitoring devices are precise. Selection biases occur due to the maladjustments of engineers. A greater description of our assumption is presented later in section 2. Secondly, the hidden Markov model in our paper is developed for discrete space, while in Kalman filter models; the state-space is continuous. Additionally, the hidden Markov model can represent an arbitrary distribution for the next value of the state variables, in contrast to the Gaussian noise model that is used in the models employing Kalman filter (Capper et al, 2005). Furthermore, our work is a significant extended work toward estimation of Markov transition probability using inspection data, which has been published in the paper of Tsuda et al, 2006b.

The following section presents a framework on selection bias and the process of deterioration with hidden condition states. Section 3 details the mathematical formulation of mixture distribution and hidden Markov transition probability. An analytical technique using Bayesian estimation and MCMC simulation is discussed in section 4. Section 5 presents an empirical study using data of the Japanese national road system. The last section summarizes the contributions of the paper and includes suggestions for future research.

\section{Selection bias and hidden condition states}

\subsection{Selection bias and representation matter}

This section details the problem of having measurement errors as selection bias in monitoring data. Based on this discussion, we establish a theoretical base for using the hidden Markov model to predict the deterioration of an infrastructure system. Fig. 1 shows monitoring data regarding the deterioration process of a road system, information which is used later in our empirical analysis. The dots in the figure represent the relation of rutting amount measured at two inspection times $\tau_{A}$ and $\tau_{B}\left(\tau_{A}<\tau_{B}\right)$ in the same road area. It is noted that road characteristics and inspection intervals vary a great deal with each sample. This fact implies that heterogeneity of the system is considered in our study. However, in the same figure, between the two inspection times, there are samples of the road sections where preventive maintenance was not performed. As long as no preventive maintenance is performed on the road, the condition of the road section cannot improve with the progress of time. Therefore, all dots reflecting values of measurement samples should lie above the 45-degree line. However, as shown in the figure, there are many samples positioned below the 45-degree line, indicating that there is considerable selection biases in the condition measurement data for the road.

\section{\{Fig. 2 is about here\}}

It is important from the outset of this paragraph to emphasize the assumption in our paper "results from observations carried out by monitoring devices are precise". And thus, our model is developed to deal with the measurement errors referred as selection biases. To make it more understanable, we distinguish two types of errors here: 1) random errors and 2) selection bias. Random errors result from faulty measurement by measuring devices, such as inspection cars with attached surface-monitoring equipment. Inevitably, surface-monitoring vehicles with devices measuring the deterioration of road sections are subject to errors, which can be thought to be random. Of course, errors due to special characteristics of the measurement device, or to differences between measurement days, are selection bias. It is necessary to consider the existence of selection bias by comparing macro characteristics, such as average values, on samples measured with different procedures. However, in the measurement of road deterioration, a different kind of selection bias exists, which we will call deterioration phenomena, a term which covers such matters as the rutting, cracking, and surface irregularity occurring with deterioration. The road condition of a certain road area is defined by the deterioration phenomena that exist within that area. However, there are numerous deterioration phenomena in a road area, and information on all such phenomena cannot be obtained by road surface inspection. Road condition is therefore defined by deterioration phenomena randomly selected among 
all existing deterioration phenomena. In this case, with two measurement times, it is possible that a deterioration phenomenon sample measured a second time shows less deterioration than when measured the first time (the condition has improved before the second measurement). However, unless preventive maintenance has been performed, the deterioration phenomena that were measured in the first time still exist without being improved, and the condition has not improved. In this case, when defining the condition of the road area, it is important to determine what deterioration phenomena have been selected. Selection biases occur when the condition of infrastructure is defined with different deterioration phenomena, and we refer to this as the problem of "representation matter."

In the evaluation of infrastructure, the deterioration condition is often defined by phenomena whereby deterioration has most progressed. For example, with the empirical analysis in this study, three parts of the same road area were measured, and the condition of parts with the most deterioration was used to represent the condition of the road area as a whole. Or, sometimes the road area is fragmented into multiple areas, and the condition of the most deteriorated part of each fragmented area is measured, and then the average of these measurements is used to define the condition of the overall area. Another example of selection bias can be summarized in three following points: 1) engineers often choose different locations of a road section for uncover its condition state. However, they do not know exactly where is the location in that road section with worst condition state; 2) after inspections, for example, on two points (A and B), they compare the deterioration between the two points and choose the worst deteriorated one as representative; 3 ) in the next inspection period, on the same road section, there is no guarantee that data from exact locations like in previously inspection is selected.

When the condition is defined with these above mentioned methods, theoretically, the condition cannot improve over time. However, data obtained by surface condition inspection is from samples selected from deterioration phenomena, and cannot be the deterioration phenomena with the most deterioration. Therefore, the condition defined by deterioration phenomena randomly selected has the selection bias of having less deterioration than the true condition measurement. In this study, we develop a hidden Markov model that takes selection biases into account, based on measurement data of road sections, and then empirically analyze the existence of system selection bias in road measurements. Furthermore, in practice, deterioration curves are often estimated by deleting the samples below the 45-degree line in Fig. 1, using samples that are positioned above the 45-degree line. However, this does not solve the problem of selection bias due to representation matter.

\subsection{Selection biases and the Hidden Markov Deterioration Process}

With Fig. 2, we explain the problem that arises when there are selection biases in condition measurements. Let us say the true deterioration of infrastructure can be expressed with $I$ rating indicators (called condition state in this study) $i(i=1, \ldots, I)$. If $i=1$, the condition is best, and if $i=I$, it is the most deteriorated. Now, assume that the result of a condition measurement taken at time $\tau_{A}$ shows the condition to be $m\left(\tau_{A}\right)=m(m=1, \ldots, I)$. However, selection biases might be included in the measurement results. We express the true condition (with no selection biases) of time $\tau_{A}$ as $m^{*}\left(\tau_{A}\right)=i(i=1, \ldots, I)$. If selection biases exist in the condition inspection results, there is no guarantee that the "observed condition" $m\left(\tau_{A}\right)=m$ is the same as the "true condition" $m *\left(\tau_{A}\right)=i$, and also there is the possibility that a value representing better condition state than the true condition state will be selected. Under such circumstance, the performance curve (or deterioration curve) of a road section is likely to be underestimated, and therefore, resulting in the underestimation of life cycle cost. This might be contracdict with the finding of Madanat and Ben-Akiva (1994), where measruement errors have been shown to increase the life cycle cost.

Here, it is assumed that the measurement results of the condition inspection are subject to the discrete probability distribution $f_{i}\left(m \mid \alpha_{i}\right)$. However, when the true condition is $i, f_{i}\left(m \mid \alpha_{i}\right)$ is a conditional probability distribution whereby the condition is judged as $m$ (hereinafter, conditional selection bias distribution). Also, $\alpha_{i}$ is a parameter vector that characterizes the probability distribution. Next, it is assumed that measurement was performed again at time $\tau_{B}=\tau_{A}+z$, after time $z$ has passed from time $\tau_{A}$. We express the measurement result of the condition inspection as $m\left(\tau_{B}\right)=n$ and the "true condition" at time $\tau_{B}$ as $m^{*}\left(\tau_{B}\right)=j$. There is also no guarantee that these two condition measurements will be the same. From the above measurement results, the pattern of the "observed 
condition" within the period $\left[\tau_{A}, \tau_{B}\right)$ is $m \rightarrow n$. The transition pattern of the "true condition" is $i \rightarrow j$. If there are selection biases, it is impossible to know if the measured condition state at each time is the "true condition state." Therefore, it is the "observed condition" $m \rightarrow n$ that is measured with the condition inspection, and the "true condition” $i \rightarrow j$ is not measured. Based on data regarding the "observed transition pattern," even if we estimate the transition probability $\pi_{m n}$, there is no guarantee that this is the same as the transition probability $\pi_{i j}$ defined by the "true transition pattern." In this way, if there are selection biases in the measurement results, the Markov deterioration process defined by the "true condition state" is hidden behind the Markov chain defined by the "observed condition states" with selection biases.

The characteristic of this study is that we use a hidden Markov model to show the state where the Markov chain of the true deterioration process is hidden within data that include selection biases. With a hidden Markov model, it is a task simultaneously to estimate the probability $f_{i}\left(m \mid \alpha_{i}\right)$ that shows the mechanism of biases in the measurement results, and the transition probability $\pi_{i j}$ that is defined with the "true condition state." Furthermore, to be accurate, it is impossible to predict the "true conditionstate" $i$, or to know what conditional selection bias distribution the "observed condition state" $m$ came from, among the different conditional selection bias distributions $f_{i}\left(m \mid \alpha_{i}\right)(i=1, \ldots, I)$. Therefore, a methodology is needed for estimating the conditional selection bias distribution that generates the data for the "observed condition state." For this reason, the hidden Markov model estimation becomes complicated, However, we will consider an estimation method in Section 4.

\section{The hidden Markov model}

\subsection{Multi-stage Exponential Markov Hazard Model}

Deterioration of a road section can be described as evolution of transition among discrete condition state. With Markov chain model, the Markov transition probability can be described as follows:

$$
\operatorname{Prob}\left[m^{*}\left(\tau_{B}\right)=j \mid m^{*}\left(\tau_{A}\right)=i\right]=\pi_{i j}
$$

where

$$
\left.\begin{array}{l}
\pi_{i j} \geq 0(i, j=1, \square, I) \\
\pi_{i j}=0(\text { when } i>j) \\
\sum_{j=i}^{I} \pi_{i j}=1
\end{array}\right\} .
$$

Given inspection data of respective road sections at times $\tau_{A}$ and $\tau_{B}$, and the corresponding characteristic variables (eg. traffic volume, thickness of road sections, etc), the properties of Markov transition matrix $\pi_{i j}$ can be estimated through Maximum likelihood estimation approach. An explicit formula for estimating the Markov transition probabilities has been derived by Tsuda et al, 2006b. In this paper, we briefly outline the formula in Eqs. (3), (4), (5), and (6) for convenient of the readers. A greater detailed of the formulation and estimation method for Markov transition probabilities using maximum likelihood estimation approach should be referred to the original paper of Tsuda et al, 2006b.

$$
\pi_{i j}(z)=\operatorname{Prob}\left[m^{*}\left(\tau_{B}\right)=j \mid m^{*}\left(\tau_{A}\right)=i\right]=\sum_{k=i}^{j} \prod_{m=i, \neq k}^{k-1} \frac{\theta_{m}}{\theta_{m}-\theta_{k}} \exp \left(-\theta_{k} z\right),
$$

where

$$
\prod_{m=i, \neq k}^{k-1} \frac{\theta_{m}}{\theta_{m}-\theta_{k}} \exp \left(-\theta_{k} z\right)=\sum_{k=i}^{j} \prod_{m=i}^{k-1} \frac{\theta_{m}}{\theta_{m}-\theta_{k}} \prod_{m=k}^{j-1} \frac{\theta_{m}}{\theta_{m+1}-\theta_{k}} \exp \left(-\theta_{k} z\right),
$$

and 


$$
\left\{\begin{array}{ll}
\prod_{m=i}^{k-1} \frac{\theta_{m}}{\theta_{m}-\theta_{k}}=1 & (k=i) \\
\prod_{m=k}^{j-1} \frac{\theta_{m}}{\theta_{m+1}-\theta_{k}}=1 \quad(k=j)
\end{array}(i=1, \square, I-1 ; j=i+1, \square, I) .\right.
$$

Transition probability from condition state $i$ to absorbing condition state $I$ is eventually defined in the following equation:

$$
\pi_{i I}(z)=1-\sum_{j=i}^{I-1} \pi_{i j}(z)(i=1, \square, I-1) .
$$

In the above equations, $\theta_{i}$ is referred as hazard rate and $z$ is time interval between the two inspections. The hazard rate $\theta_{i}$ is a representation of hazard function, which is defined as conditional probability, a ratio of probability density function of transition of condition state $i$ (numerator) and survival probability function (denominator). In the cited paper, the hazard function is assumed to follow exponential function form. A detail of exponential hazard function and its mathematical derivation should be referred to original paper.

The hazard rate can be expressed by multiplicative form of characteristic variable $x$ and unknown parameter $\beta_{i}^{\prime}$ (Lancaster, 1990). The unknown parameter $\beta_{i}^{\prime}$ is the target of estimation in statistical models, and it is often referred as model's parameter.

$$
\theta_{i}=\theta_{i}(x)=x \beta_{i}^{\prime}
$$

Statistically, the characteristic variable $x$ in Eq. (7) is often referred as model's covariates or variables. This characteristic vector genuinely represents the heterogeneity factors in our model. Example of the characteristic variables are daily or annual traffic volumes, ambient temperatures, thickness of road sections, etc. Such characteristic variables are the factors affecting the deterioration process. For instance, road sections engaging with a high number of daily traffic volume oftenly have faster deterioration than that engaging with less daily traffic volume. The values of characteristic variables of different road sections are different, and therefore we considere them as observable heterogeneity factors.

\subsection{Mixture distribution mechanism}

This section explains the mathematical formulation of the hidden Markov model based on the mixture distribution mechanism. Assumptions are as stated in Section 2. In fact, it is uncertain whether the probability distribution function $f_{i}\left(m \mid \alpha_{i}\right)(i=1, \square, I)$ can be used to estimate the true condition state $i$. However, we are able to express the probabilistic dependence of observed condition state $m\left(\tau_{A}\right)=m$ on true condition state $i$ by means of the likelihood function $f_{i}\left(m \mid \alpha_{i}\right)(i=1, \square, I)$.

$$
\left.\square\left(\tau_{A}\right)=m\right)=\sum_{i=1}^{I} \pi_{i}\left(\tau_{A}\right) f_{i}\left(m \mid \alpha_{i}\right),
$$

where $\pi_{i}\left(\tau_{A}\right)$ is the probability of true condition state $i$ at inspection time $\tau_{A}$. Eq. (8) depicts the conditional probability distribution of observed condition state $m\left(\tau_{A}\right)=m$ on true condition state $i$. In other words, it portrays the conditional probability distribution of observed condition state $m\left(\tau_{A}\right)=m$ by averaging the distributed values of selection biases over the range of true condition states. Models which mix mechanisms of selection biases are referred to as mixture distribution models.

Similarly, the probability distribution of observed condition states at inspection time $\tau_{B}=\tau_{A}+z\left(\tau_{A}<\tau_{B}\right)$ can be described with mixed forms. The likelihood function $\square\left(m\left(\tau_{B}\right)=n\right)$, to which observed condition state $m\left(\tau_{B}\right)=n$ 
at inspection time $\tau_{B}$ can be defined as

$$
\ell\left(m\left(\tau_{B}\right)=n\right)=\sum_{i=i}^{I} \pi_{i}\left(\tau_{A}\right) \sum_{j=i}^{I} \pi_{i j}(z) f_{j}\left(n \mid \alpha_{j}\right)
$$

As a result, the likelihood function $\square\left(m\left(\tau_{A}\right)=m, m\left(\tau_{B}\right)=n\right)$, at which we observe condition state $m\left(\tau_{A}\right)=m$ at inspection time $\tau_{A}$ and condition state $m\left(\tau_{B}\right)=n$ at inspection time $\tau_{B}$, can be defined as follows:

$$
\left.\square\left(\tau_{A}\right)=m, m\left(\tau_{B}\right)=n\right)=\sum_{i=1}^{I} \pi_{i}\left(\tau_{A}\right) f_{i}\left(m \mid \alpha_{i}\right)\left(\sum_{j=i}^{I} \pi_{i j}(z) f_{j}\left(n \mid \alpha_{j}\right)\right) .
$$

It is noted that the random variables $m\left(\tau_{A}\right)$ and $m\left(\tau_{B}\right)$ (as well as $m^{*}\left(\tau_{A}\right)$ and $m^{*}\left(\tau_{B}\right)$ ) are correlated through the Markovian transition probability. The probability distribution functions $f_{i}\left(m \mid \alpha_{i}\right)$ and $f_{j}\left(n \mid \alpha_{j}\right)$ are independent of each other. In other words, the distribution of observed condition states depends on hidden characteristics or selection biases at respective inspection times $\tau_{A}$ and $\tau_{B}$.

\subsection{Initial values of the condition states}

As can be seen from Eq. (10), there are three unknown components: the initial distribution $\pi_{i}\left(\tau_{A}\right)$, the probability distribution function $f_{i}\left(m \mid \alpha_{i}\right)$, and the Markov transition probability $\pi_{i j}(z)$. The value of the initial probability distribution $\pi_{i}\left(\tau_{A}\right)$ is regarded as transcendental information. The initial probability distribution can be assumed as a variable of non-parametric distribution. However, assuming it to be a non-parametric variable limits the study for a large number of monitoring data since characteristic variables concerning one road section do not share the same values with those of other road sections. It is therefore advisable to determine the initial value of the condition state immediately after $M \& R$ activities, when a road section is renewed to a good condition state whereby $i=1$. For example, if $M \& R$ action is carried out just before time $\tau_{0}$, the initial probability distribution can be defined as

$$
\pi\left(\tau_{0}\right)=\left\{\pi_{1}\left(\tau_{0}\right), \square, \pi_{I}\left(\tau_{0}\right)\right\}=(1,0, \square, 0) .
$$

Evidently, properties of the vector $\pi\left(\tau_{0}\right)$ are measurable. Thus, if $M \& R$ actions are implemented at alternative times $\tau_{1}, \square, \tau_{T}$, the initial value of probability distribution $\pi_{i}\left(\tau_{A}\right)$ can also be defined. To come up with a general likelihood function for the conditional probability distribution of observed condition states $m$, observed condition states after $M \& R$ actions at times $\tau_{t}(t=1, \ldots, T)$ are assumed as $m\left(\tau_{t}\right)=m_{t}$. Durations between two consecutive $M \& R$ actions from $t-1$ to $t$ are denoted as $z_{t}(t=1, \square, T)$. As a result, likelihood function $L(\alpha, m, z)$, which describes the conditional probability distribution of observed condition states $m=\left(m_{1}, \square, m_{T}\right)$, can be recurrently defined:

$$
\begin{aligned}
& L(\alpha, m, z)=\sum_{j=1}^{I} \pi_{1 j}\left(z_{1}\right) f_{j}\left(m_{1} \mid \alpha_{j}\right) \ell_{j}\left(m_{1}\right), \\
& \ell_{h}\left(m_{t}\right)=\sum_{j=h}^{I} \pi_{h j}\left(z_{t}\right) f_{j}\left(m_{t} \mid \alpha_{j}\right) \ell_{j}\left(m_{t+1}\right) \quad(1 \leq t \leq T-1), \\
& \ell_{h}\left(m_{T}\right)=\sum_{j=h}^{I} \pi_{h j}\left(z_{T}\right) f_{j}\left(m_{T} \mid \alpha_{j}\right) .
\end{aligned}
$$


The model's parameters to be estimated are $\beta$ and $\alpha$, which are embedded in the Markov transition probability $\pi_{i j}$ through Eq. (3) , Eq. (7) and the probabilitity mass function $f(\cdot)$ respectively. ikelihood functions in Eq. (12), Eq. (13), and Eq. (14). The maximum likelihood estimation method can be used to estimate the values of a model's parameters based on numerical analysis with an objective likelihood function. However, the method is limited in that it requires a high order of derivative and a high degree of computation for solving the optimal condition of nonlinear polynomial equations. Therefore, within the estimation framework for the hidden Markov model, the maximum likelihood method is not the preferred approach (Titterington et al., 1985). Attempts to overcome the limitations of the maximum likelihood method by using Bayesian estimation have been proposed in a great number of advance statistic literatures (e.g. Geman and Geman 1984; Scott, 2000; Jeff, 2006).

\subsection{Complete likelihood function}

Distribution of selection biases is assumed by means of a hidden variable $s=\left(s_{0}, \square, s_{T}\right)$. If there is no $M \& R$ action in the inspection period, the following condition is satisfied:

$$
s_{0}=1 \leq s_{1} \leq \square \leq s_{T} \leq I \text {. }
$$

Furthermore, if the hidden variable is measurable, its value can be used to update the probability distribution of true condition state $i$, which is hidden because of selection biases. In addition, to identify the possibility of measurement of the hidden variable, a dummy variable $\delta$ is assigned with the following conditions:

$$
\delta_{t i}=\left\{\begin{array}{ll}
1 & s_{t}=i \\
0 & s_{t} \neq i
\end{array}(t=1, \square, T ; i=1, \square, I) .\right.
$$

With this assumption and according to Dempster et al, 1977, the likelihood functions in (12), (13), and (14) are then described as follows:

$$
\begin{aligned}
L(s, \alpha, m, z) & =\prod_{i=1}^{I}\left\{\pi_{1 i}\left(z_{1}\right)^{\delta_{1 i}} f_{i}\left(m_{1} \mid \alpha_{i}\right)^{\delta_{1 i}} \prod_{t=2}^{T} \prod_{j=i}^{I} \pi_{i j}\left(z_{t}\right)^{\delta_{t-1 i} \delta_{t j}} f_{j}\left(m_{t} \mid \alpha_{j}\right)^{\delta_{t j}}\right\} \\
& =\prod_{t=1}^{T}\left\{\pi_{s_{t-1} s_{t}}\left(z_{t}\right) f_{s_{t}}\left(m_{t} \mid \alpha_{s_{t}}\right)\right\}=\prod_{t=1}^{T} \pi_{s_{t-1} s_{t}}\left(z_{t}\right) \prod_{t=1}^{T} f_{s_{t}}\left(m_{t} \mid \alpha_{s_{t}}\right) .
\end{aligned}
$$

In Bayesian statistics, Eq. (17) is commonly referred to as a complete likelihood equation, with a finer explicit form than that in likelihood Eqs. (12), (13), and (14). Nevertheless, a difficulty remains at this point in how to assign a realistic value for the hidden variable $s$, since it is unobservable. In view of probability distribution, hidden variable $s$ can be derived when full conditional posterior distribution with Bayesian inference is applied. The prior probability distribution in the Bayesian estimation is assumed as follows:

$$
\operatorname{Prob}\left\{s_{t}=i \mid s_{-t}, \alpha, \xi\right\}=\frac{t\left(s_{-t}^{i}, \alpha, m, z\right)}{\sum_{i=s_{t-1}}^{s_{t+1}} L\left(s_{-t}^{i}, \alpha, m, z\right)}=\frac{\omega_{i t} f_{i}\left(m_{t} \mid \alpha_{i}\right)}{\sum_{j=s_{t-1}}^{s_{t+1}} \omega_{j t} f_{j}\left(m_{t} \mid \alpha{ }_{j}\right)},
$$

where $s_{-t}=\left(s_{1}, \square, s_{t-1}, s_{t+1}, \square, s_{T}\right), s_{-t}^{i}=\left(s_{1}, \sqcup, s_{t-1}, i, s_{t+1}, \square, s_{T}\right)$, and $s_{t}=i\left(i \in\left\{s_{t-1}, \square, s_{t+1}\right\}\right)$. In addition, $\omega_{j t}$ satisfies 


$$
\omega_{j t}= \begin{cases}\pi_{1 j} \pi_{j s_{2}} & t=1 \\ \pi_{s_{t-1} j} \pi_{j s_{t+1}} & 2 \leq t \leq T . \\ \pi_{s_{T-1} j} & t=T\end{cases}
$$

If the posterior probability distribution of hidden variable $s_{t} \in\left\{s_{t-1}, \square, s_{t+1}\right\}$ at time $t$ is measurable, transition probability $\pi_{i j}(z)(i=1, \square, I ; j=i, \square, I)$ and probability distribution function $f_{i}\left(m \mid \alpha_{i}\right)(i=1, \square, I)$ can ultimately be estimated. It is also noted that the posterior probability distribution of hidden variable $s_{t} \in\left\{s_{t-1}, \square, s_{t+1}\right\}$ is conditionally dependent on the observed value of $s_{-t}$.

To solve the likelihood function in Eq. (18), it is necessary to estimate the value of hidden variable $s$. The main task, therefore, is to estimate the two unknown parameters $\alpha$ and $\beta$, which are embedded in the transition probability functions. In reality, the possibility of seeking the posterior distribution of all hidden variables is very hard. Thus, it is recommended that MCMC simulation be used in randomly generating hidden variable $s$.

\subsection{Conditional distribution of selection biases}

As mentioned in section 2, the representative condition state of a road section generally happens to be the worst condition state among several observed on the same section. Thus, it is possible to assume the range of observed condition state $m$ in a domain $m(m=1, \ldots, i)$. The relationship between observed condition state $m$ and true condition state $i$ implies selection biases on the same road section. Observed condition states can be considered to be chosen through a random selection process. However, probabilistic inference on the value of probability distribution function $f_{i}\left(m \mid \alpha_{i}\right)(m=1, \square, i)$ is a matter of some difficulty. In this research, the distribution probability function $f_{i}\left(m \mid \alpha_{i}\right)(m=1, \square, i)$ is assigned to satisfy the following conditions:

$$
f_{i}\left(m \mid \alpha_{i}\right)=\left\{\begin{array}{lll}
0 & \text { when } & m>i \\
\alpha_{m}^{i} & \text { when } & m \leq i
\end{array},\right.
$$

where parameter $\alpha_{m}^{i}$ is assumed as a non-parametric constant satisfying

$$
\begin{aligned}
& 0 \leq \alpha_{m}^{i} \leq 1, \\
& \sum_{m=1}^{i} \alpha_{m}^{i}=1 .
\end{aligned}
$$

The probability distribution of parameter $\alpha_{m}^{i}$ can be estimated if the quantity of monitoring data is sufficient. This is a non-parametric approach in cases where there is no prior information regarding selection biases. Moreover, the assumption in Eq. (20) reflects management practice and also eliminates the so-called "label switching" problem in hidden Markov model estimation (Zucchini and MacDonald,2009; Scott, 2000).

\section{Estimation methodology}

\subsection{Markov Chain Monte Carlo (MCMC) method}

In statistics with Bayesian inference, prior and posterior probability are employed with the aim of estimating the values of a model's parameters. However, in hazard analysis, it is difficult to define the prior probability distribution, even in a simple condition states deterioration model. Methods to overcome the problems in the assumption of prior probability distribution often require numerical analyses with multi-dimensional integration, and this necessity remains a limitation of Bayesian estimation. 
In recent years, an appealing solution to the problem in Bayesian estimation has been proposed, with the application of MCMC simulation. The MCMC simulation technique is attractive because it requires neither a high level of derivative nor multi-dimensional integration of a model's objective functions. Estimation results in a great deal of applied statistics research have been improved through a combination of Bayesian estimation and MCMC simulation.

In MCMC simulation, Gibbs sampling and Metropolis Hastings (Metropolis-Hastings or MH) techniques have been extensively discussed. Our list of references includes a good example of the use of MCMC simulation in research related to image restoration. In the cited study, the algorithm of Gibbs sampling was used to estimate the posterior distribution in Bayesian estimation. In $\mathrm{MH}$ law, the iterative parameter $\beta$ is defined by repeatedly generating random numbers through the conditional probability density function. In the present research, we propose an extended estimation methodology to estimate the parameters of the hidden Markov model based on the literature regarding Bayesian estimation for the Weibull hazard model of Tsuda et al. (2006a).

To estimate further the parameters in hidden Markov models, an analytical approach using method of maximum likelihood has already been shown to have limitations (Titterington et al., 1985; Robert, 1996). Since the hidden Markov model is considered as one type of mixture distribution model, a great deal of research has suggested defining a set of complete likelihood functions instead of using conventional likelihood functions (Diebolt and Robert, 1994; Robert et al., 2000). In view of MCMC simulation, it is necessary to develop an explicit algorithm for estimating the Markov transition probability with multi-condition states. In this research, we propose an analytical approach using Bayesian estimation and MCMC simulation for estimating the Markov transition probability of the multi-stage exponential Markov model, which is briefly presented in section 3.1.

\subsection{Formulation of the model}

Visual inspection is carried out on each section $k$ of the entire road system (with $K$ as the total number of road sections). The observed data on each section over a time-series can be denoted as $\tau_{t}^{k}\left(t=1, \square, T^{k}\right)$, with $T^{k}$ as the number of inspection times for road section $k$. Each observed condition state from the visual inspection is represented as $\bar{m}\left(\tau_{t}^{k}\right)$, with the sign - indicating measurable data. $\bar{\xi}=\left(\bar{\xi}^{1}, \square, \bar{\xi}^{K}\right)$ is denoted as the vector of measurable data concerning $\sum_{k=1}^{K} T^{k}$ number of records.

The deterioration process of a road section is influenced by the changes in values of characteristic variables such as traffic volume, thickness of overlay, weather, etc. The values of characteristic variables are recorded and stored as monitoring data. To consider the effects of characteristic variables on deterioration, vector $\bar{x}_{t}^{k}$ is assumed to represent characteristic variables. In addition, duration between two consecutive visual inspections is defined as $\bar{z}_{t}^{k}=\tau_{t}^{k}-\tau_{t-1}^{k}$. In summary, observed information concerning each section of road can be symbolized as $\bar{\xi}_{t}^{k}=\left(\bar{m}_{t}^{k}, \bar{z}_{t}^{k}, \bar{x}_{t}^{k}\right)$, with $m\left(\tau_{t}^{k}\right)=\bar{m}_{t}^{k}$. As a result, simultaneous probability distribution for the entire $K$ samples can be defined as follows:

$$
L(\alpha, m, \beta, \bar{\xi})=\prod_{k=1}^{K}\left\{\prod_{t=1}^{T^{k}} \pi_{s_{t-1}^{k} s_{t}^{k}}\left(\bar{z}_{t}^{k}\right) \prod_{t=1}^{T^{k}} f_{s_{t}^{k}}\left(\bar{m}_{t}^{k} \mid \alpha_{s_{t}^{k}}\right)\right\}=\prod_{k=1}^{K}\left[\prod_{t=1}^{T^{k}} \alpha_{\bar{m}_{t}^{k}}^{s_{t}^{k}} \sum_{l=s_{t-1}^{k}}^{s_{t}^{k}}\left\{\prod_{i=s_{t-1}^{k}, \neq l}^{l-1} \frac{\theta_{i}^{k}}{\theta_{i}^{k}-\theta_{l}^{k}} \exp \left(-\theta_{l}^{k} \bar{z}_{t}^{k}\right)_{\mid}^{k}\right\}_{\}}\right\}
$$

In likelihood Eq. (23), theoretically, hazard function can be described either by using exponential form as $\theta_{i}^{k}=\exp \left(x^{k} \beta_{i}^{\prime}\right)$ or by plain form (refer to Eq. (7)). In order to estimate unknown parameters and hidden variables (selection biases), we need to propose a feasible numerical method to solve the likelihood function in Eqs. (12), (13), and (14). By solving Eq. (23), the values of $\alpha=\left(\alpha_{1}, \square, \alpha_{I-1}\right), \beta=\left(\beta_{1}, \square, \beta_{I-1}\right)$, and hidden variable $s=\left(s^{1}, \square, s^{K}\right)$ can be obtained. If parameter vectors $\alpha$ and $\beta$ are known, the posterior distribution of hidden variable $s_{t}^{k}\left(t=1, \square, T^{k} ; k=1, \square, K\right)$ can be estimated as well. Given the condition $s_{-t}^{k}=\left(s_{1}^{k}, \square, s_{t-1}^{k}, s_{t+1}^{k}, \square, s_{T^{k}}^{k}\right)$, the conditional probability, in which hidden variable $s_{t}^{k}\left(s_{t}^{k} \in\left\{s_{t-1}^{k}, \square, s_{t+1}^{k}\right\}\right)$ equals true condition state $i$, is finally estimated: 


$$
\operatorname{Prob}\left\{s_{t}^{k}=i \mid s_{-t}^{k}, \alpha, \xi\right\}=\frac{\omega_{i t}^{k} f_{i}\left(m_{t}^{k} \mid \alpha_{i}\right)}{\sum_{j=s_{t-1}^{k}}^{k} \omega_{j t}^{k} f_{j}\left(m_{t}^{k} \mid \alpha_{j}\right)},
$$

\subsection{Bayesian estimation}

As a common practice in Bayesian estimation, the assumptions for prior probability distribution of parameters $\alpha$ and $\beta$ should be based on various sources of prior experience information (Jeff, 2006). Any new information concerning monitoring data $\xi$ shall be directly used for the estimation of likelihood function $L(\alpha, \beta, \bar{\xi})$. The updating rule in Bayesian estimation constantly improves the level of accuracy for prior probability distribution of parameters. By using the most up-to-date monitoring data, values of parameters $\alpha$ and $\beta$ specifying the probability density function $\rho(\alpha, \beta \mid \xi)$ can be simultaneously obtained. However, according to Ibrahim et al. (2001), a single time of assuming the prior probability density function cannot guarantee the accuracy of estimation results since prior probability density function can be assumed in various ways. Thus, it is advisable to define prior probability density function concomitantly with visual inspections. As a rule of thumb, the influence of the prior probability density function will gradually decrease as the number of monitoring data increases.

As mentioned in Section 3.5, constant parameter $\alpha_{i}=\left(\alpha_{1}^{i}, \square, \alpha_{i}^{i}\right)$ in Eq. (20) is assumed to satisfy the conditions in Eq. (21) and Eq. (22). On that account, we introduce conjugate Dirichlet distribution for the prior probability density function of constant $\alpha_{i}$ :

$$
\begin{aligned}
& \eta_{i}\left(\alpha_{i} \mid v^{i}\right)=\Psi_{i}\left(v^{i}\right) \prod_{m=1}^{i}\left(\alpha_{m}^{i}\right)^{v_{m}^{i}-1} \\
& \Psi_{i}\left(v^{i}\right)=\frac{\Gamma\left(v_{1}^{i}+\square+v_{i}^{i}\right)}{\Gamma\left(v_{1}^{i}\right) \square \Gamma\left(v_{i}^{i}\right)} \text { and } \sum_{m=1}^{i} \alpha_{m}^{i}=1
\end{aligned}
$$

It is noted that Direclet distribution infers a constant parameter $v^{i}=\left(v_{1}^{i}, \square, v_{i}^{i}\right)$, which spontaneously satisfies the constant parameter $\alpha_{i}$ in (21) and (22).

The assumption for the prior probability density function of parameter $\beta_{i}$ can be defined in the next step. The conjugate multidimensional normal distribution $\beta_{i}: N_{M}\left(\zeta_{i}, \Sigma_{i}\right)$ is assumed for the prior probability density function in $M$ dimension:

$$
h\left(\beta_{i} \mid \zeta_{i}, \Sigma_{i}\right)=\frac{1}{(2 \pi)^{\frac{M}{2}} \sqrt{\left|\Sigma_{i}\right|}} \cdot \exp \left\{-\frac{1}{2}\left(\beta_{i}-\zeta_{i}\right) \Sigma_{i}^{-1}\left(\beta_{i}-\zeta_{i}\right)^{\prime}\right\},
$$

where $\Sigma_{i}$ and $\zeta_{i}$ of $N_{M}\left(\zeta_{i}, \Sigma_{i}\right)$ are covariance matrix and standard covariance of the prior distribution respectively. As a result, a proportional result of the probability density function $\rho(\alpha, \beta \mid s, \xi)$ can be re-formulated:

$$
\begin{aligned}
& \rho(\alpha, \beta \mid s, \xi) \propto L(\alpha, \beta, s, \xi) \prod_{i=1}^{I-1}\left\{h\left(\beta_{i} \mid \mu_{i}, \Sigma_{i}\right) \eta_{i}\left(\alpha_{i} \mid v^{i}\right)\right\} \\
& \propto \prod_{k=1}^{K}\left[\prod_{t=1}^{T^{k}} \sum_{l=s_{t-1}^{k}}^{s_{t}^{k}}\left\{\prod_{i=s_{t-1}^{k}, \neq l}^{l-1} \frac{\theta_{i}^{k}}{\theta_{i}^{k}-\theta_{l}^{k}} \exp \left(-\theta_{l}^{k} z_{t}^{k}\right)\right\} \cdot \prod_{i=1}^{I-1} \exp \left\{-\frac{1}{2}\left(\beta_{i}-\zeta_{i}\right) \Sigma_{i}^{-1}\left(\beta_{i}-\zeta_{i}\right)^{\prime}\right\}\right.
\end{aligned}
$$




$$
\cdot\left(\prod_{t=1}^{T^{k}} \alpha_{\bar{m}_{t}^{k}}^{s_{t}^{k}}\right)\left(\prod_{i=1}^{I} \prod_{m=1}^{i}\left(\alpha_{m}^{i}\right)^{v_{m}^{i}-1}\right)_{j}^{j}
$$

\subsection{Gibbs sampling}

A direct estimation of probability density function $\rho(\alpha, \beta \mid \xi)$ in hidden Markov models is impractical. By using the MCMC simulation, value samples (specimens) of parameters $\alpha$ and $\beta$ can be alternately extracted from the probability density function (Geman and Geman, 1984). In Eq. (27), parameters $\alpha$ and $\beta$ can be mutually used to express the probability density function. Approximation of $\rho(\alpha \mid s, \xi)$ and $\rho(\beta \mid s, \xi)$ can be further described as follows:

$$
\begin{aligned}
& \rho(\alpha \mid s, \xi) \propto\left(\prod_{k=1}^{K} \prod_{t=1}^{T^{k}} \alpha_{\bar{m}_{t}^{k}}^{s_{t}^{k}}\right)\left\{\prod_{i=1}^{I} \prod_{m=1}^{i}\left(\alpha_{m}^{i}\right)^{v_{m}^{i}-1}\right\}, \\
& \rho(\beta \mid s, \xi) \propto\left\{\prod _ { k = 1 } ^ { K } \prod _ { t = 1 } ^ { T ^ { k } } \sum _ { l = s _ { t - 1 } ^ { k } } ^ { s _ { t } ^ { k } } \left[\left.\left.\prod_{i=s_{t-1}^{k}, \neq l}^{l-1} \frac{\theta_{i}^{k}}{\theta_{i}^{k}-\theta_{l}^{k}} \exp \left(-\theta_{l}^{k} z_{t}^{k}\right)\right|_{\downarrow}\right|_{l} \prod_{i=1}^{I-1} \exp \left\{-\frac{1}{2}\left(\beta_{i}-\zeta_{i}\right) \Sigma_{i}^{-1}\left(\beta_{i}-\zeta_{i}\right)^{\prime}\right\} .\right.\right.
\end{aligned}
$$

The conditional posterior distribution of hidden variable $S$ can be expressed in Eq. (24). A detailed procedure of the analytical approach using Bayesian estimation and MCMC simulation is given in the following steps.

\subsubsection{Step 1: Initial parameter values}

Parameter vectors $v^{i}(i=1, \square, I), \zeta_{i}$, and $\Sigma_{i}(i=1, \square, I-1)$ of the prior probability distribution in Eq. (25) and Eq. (26) have an arbitrary set of values. The value of the hidden variable $s^{(0)}=\left(s^{(1,0)}, \square, s^{(K, 0)}\right)$ is initially chosen so as to satisfy $s^{(k, 0)}=\left(s_{1}^{k, 0}, \square, s_{T}^{k, 0}\right), 1 \leq s_{1}^{k, 0} \leq \square \leq s_{T}^{k, 0} \leq I$, and $m_{t}^{k} \leq s_{t}^{k, 0}(t=1, \square, T ; k=1, \square, K)$. The influence of initial values $\alpha^{(0)}$ and $\beta^{(0)}$ gradually becomes weaker as more information generated by MCMC simulation is accumulated. To begin with the iteration, a sampling number $n$ in MCMC simulation is assigned as $n=1$.

\subsubsection{Step 2: Sampling parameter $\alpha^{(n)}$}

This section describes the estimation of $\alpha^{(n)}=\left(\alpha_{1}^{(n)}, \square, \alpha_{I-1}^{(n)}\right)$ based on prior hidden variable $s^{(n-1)}$. The probability density function $\rho\left(\alpha^{(n)} \mid s^{(n-1)}, \xi\right)$ in Eq. (28) can be rewritten, with an extended description $\alpha_{i}^{(n)}$ $=\left(\alpha_{m}^{i, n}: m=1, \square, i\right)$ :

$$
\notin\left(\alpha_{i}^{(n)} \mid S^{(n-1)}, \xi\right) \propto\left\{\prod_{k=1}^{K} \prod_{t=1}^{T^{k}} \alpha_{m_{t}^{k}}^{s_{t}^{k,(n-1)}} \mid\right\}\left\{\left\{\prod_{m=1}^{i}\left(\alpha_{m}^{i, n}\right)^{v_{m}^{i}{ }^{i}}\right\}=\prod_{m=1}^{i}\left(\alpha_{m}^{i, n}\right)^{v_{m}^{i}+N_{m}^{i,(n-1)}-1},\right.
$$

where $N_{m}^{i,(n-1)}$ is defined in the following equation, particularly when values of condition state $\bar{m}$ and hidden variable $s^{(n-1)}$ are available:

$$
N_{m}^{i,(n-1)}=\#\left\{\bar{m}_{t}^{k}=m \cap s_{t}^{k,(n-1)}=i\right\}
$$

Indication $\#\{\}$ in Eq. (31) presents the number of measurable samples, to which the equation in the parentheses \{\} refers. Parameter $\alpha$ in Eq. (30) is assumed to follow Dirichlet distribution, with its parameter as $v_{m}^{i}+N_{m}^{i,(n-1)}-1$. 
The parameters of Dirichlet distribution are subsequently updated by using extracted samples $\alpha_{i}^{(n)}=\left(\alpha_{1}^{i,(n)}, \square, \alpha_{i}^{i,(n)}\right)$ through Gibbs sampling. It is noted that samples of parameter $\alpha_{i}^{(n)}$ are evaluated from the entire range of condition state $i(i=1, \ldots, I)$.

\subsubsection{Step 3: Sampling parameter $\beta^{(n)}$}

This section describes an algorithm for estimating unknown parameter $\beta$ of the multi-stage exponential Markov model. Additional notation of the unknown parameter is $\beta_{-e q}$. It is noticed from the notation that element $\beta_{e q}$ $(e, q)(e, q=1, \square, M)$ is excluded from the list of unknown parameter $\beta$. Thus, we formulated the conditional probability density function $\rho\left(\beta_{e q} \mid \beta_{-e q}, s, \xi\right)$ of $\beta_{e q}$ based on the assumed value of $\beta_{-e q}$ in Eq. (29):

$$
\begin{gathered}
\hat{\rho}\left(\beta_{e q} \mid \beta_{-e q}, s, \xi\right) \propto \prod_{i=1}^{e} \prod_{j=e}^{I} \prod_{k=1}^{K} \prod_{t=1}^{T^{k}}\left\{\prod_{l=i}^{j-1}\left(\theta_{l}^{k}\right)^{\delta_{i j}^{t k}-\delta_{i e}^{t k}} \sum_{h=i}^{j} \cdot \prod_{l=i, \neq h}^{h-1} \frac{1}{\theta_{l}^{k}-\theta_{h}^{k}} \exp \left(-\theta_{h}^{k} z_{t}^{k}\right)\right\} \\
\cdot \prod_{i=1}^{I-1} \exp \left\{-\frac{1}{2}\left(\beta_{i}-\zeta_{i}\right) \Sigma_{i}^{-1}\left(\beta_{i}-\zeta_{i}\right)^{\prime}\right\} \\
\propto \prod_{i=1}^{e} \prod_{j=e}^{I} \prod_{k=1}^{K} \prod_{t=1}^{T^{k}}\left[\left.\prod_{l=i}^{j-1}\left\{\exp \left(\beta_{e q} x_{q}^{k}\right)\right\}^{f_{i j}^{t k}-\delta_{i e}^{t k}} \sum_{h=i}^{j} \prod_{l=i, \neq h}^{h-1} \frac{1}{\theta_{l}^{k}-\theta_{h}^{k}} \exp \left(-\theta_{h}^{k} z_{t}^{k}\right)\right|^{\delta_{i j}^{t k}} \exp \left\{-\frac{\sigma_{e}^{q q}}{2}\left(\beta_{e q}-\ddot{\zeta}_{e}\right)^{2}\right\},\right. \\
\hat{\zeta}_{e}^{q}=\zeta_{e}^{q}+\sum_{h=1, \neq q}^{M}\left(\beta_{e h}-\zeta_{e}^{h}\right) \sigma_{e}^{h q},
\end{gathered}
$$

where $\delta_{i e}^{t k}$ and $\delta_{i j}^{t k}$ are dummy variables:

$$
\delta_{i e}^{t k}=\left\{\begin{array}{l}
1 \quad \text { when } s_{t-1}^{k}=i=e \\
0 \quad \text { otherwise }
\end{array} \text { and } \delta_{i j}^{t k}=\left\{\begin{array}{l}
1 \quad \text { when } s_{t-1}^{k}=i, s_{t}^{k}=j \\
0 \quad \text { otherwise }
\end{array}\right.\right.
$$

$\zeta_{e}^{q}$ and $\sigma_{e}^{h q}$ are the prior expected values of vector $\zeta_{e}$ and prior standard covariance of entire procession $\Sigma_{e}^{-1}$ with respect to condition state $q$ and $(h, q)$. In addition, $\sum_{h=1, \neq q}^{M}$ is the summation of all condition states from $\sum_{h=1, \neq q}^{M}$, excluding index $q$. Expected condition state is generated by using conditional probability density functions. By using the generated condition states, we can come up with the posterior distribution of parameter $\beta$. A detailed MCMC simulation for estimating the posterior distribution will be presented later. However, a summation of the random sampling procedure for parameter $\beta^{(n)}=\left(\beta_{11}^{(n)}, \square, \beta_{I-1 M}^{(n)}\right)$ up to this point is presented as follows:

- Step 3.1 - value of parameter $\beta_{11}^{(n)}$ is randomly generated from $\hat{\rho}\left(\beta_{11}^{(n)} \mid \beta_{-11}^{(n-1)}, s^{(n-1)}, \xi\right)$.

- Step 3.2 - value of parameter $\beta_{12}^{(n)}$ is randomly generated from $\hat{\rho}\left(\beta_{12}^{(n)} \mid \beta_{-12}^{(n-1)}, s^{(n-1)}, \xi\right)$.

- Step 3.3 - procedure similar to steps 3.1 and 3.2 is repeated.

- Step 3.4 - value of parameter $\beta_{I-1 M}^{(n)}$ is randomly generated from $\hat{\rho}\left(\beta_{I-1, M}^{(n)} \mid \beta_{-(I-1 M)}^{(n-1)},{ }^{(n-1)}, \xi\right)$.

Gibbs sampling is applied to generate condition states from $(I-1) M$ conditional posterior probability density functions. The so-called "adaptive sampling rejection" (Gilks and Wild, 1992) can be used as a technique to generate specimens of parameters in the posterior distribution, which is explained in Eq. (32).

\subsubsection{Step 4: updating hidden variable}

Given the prior value of hidden variable $s_{-t}^{k,(n-1)}=\left(s_{1}^{k, n}, \square, s_{t-1}^{k, n}, s_{t+1}^{k,(n-1)}, \square, s_{T^{k}}^{k,(n-1)}\right)$, a new hidden variable $s^{(n)}$ is 
randomly selected based on the conditional probability law in Eq. (24). Random generation applies for all condition states $s_{t}^{k, n}\left(s_{t}^{k, n} \in\left\{s_{t-1}^{k, n}, \square, s_{t+1}^{k,(n-1)}\right\}\right)$.

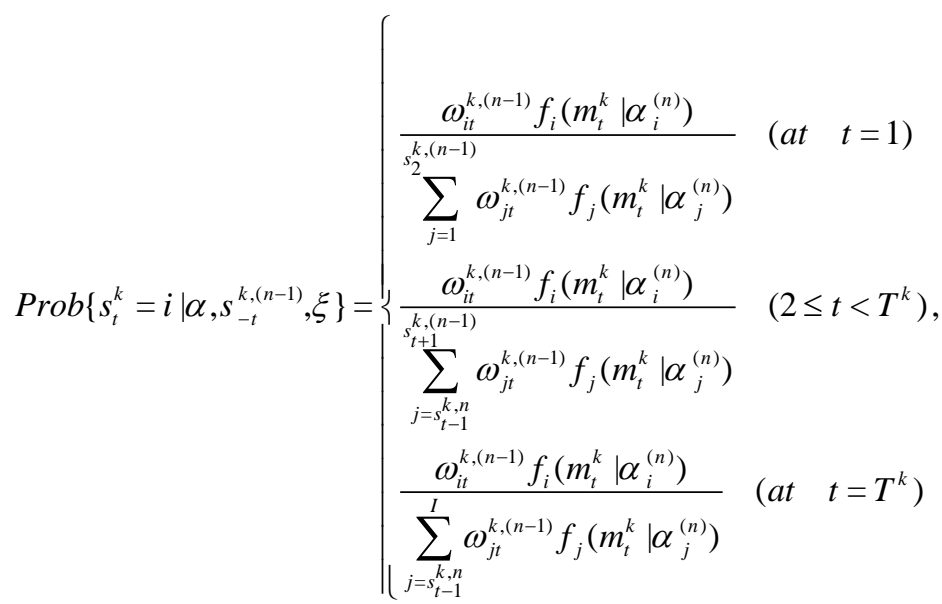

Hidden variable $s_{t}^{k, n}\left(t=1, \square, T^{k}\right)$ is estimated in succession, starting from $t=1$ for all numbers of sample $k(k=1, \square, K)$.

\subsubsection{Step 5: determining algorithm adjustment}

After step 4.4.4, the values of parameters $\alpha^{(n)}, \beta^{(n)}$, and hidden variable $s^{(n)}$ are recorded. In the next iteration $n=n+1$, the program returns to step 4.4.2. If the algorithm satisfies $n \leq \bar{n}$, the program stops.

A major concern is the number of condition states generated in simulation. The number should be carefully examined. In several cases, steady condition states could not be reached even though a large number of condition states had been accumulated. It is desirable to eliminate this problem by introducing a minimum set of parameter value as $\underline{n}$. In fact, values of parameters $\alpha^{(n)}$ and $\beta^{(n)}(n=\underline{n}+1, \underline{n}+2, \square, \bar{n})$ are embedded in the posterior probability density function $\rho(\alpha, \beta \mid \xi)$ through Gibbs sampling. As a result, estimation of the posterior distribution of parameters $\alpha, \beta$ becomes analytically feasible. To verify the estimation results, we apply the Geweke statistical test.

\subsection{Posterior distribution statistic}

Statistical testing for parameters $\alpha$ and $\beta$ can be carried out based on samples generated by using MCMC simulation. However, in the simulation, the probability density function $\rho(\alpha, \beta \mid \xi)$ cannot be considered as an analytical function. Therefore, instead of using a full parametric approach for statistical testing, a non-parametric approach is recommended. With the Gibbs sampling method, samples concerning $\theta^{(n)}=\left(\alpha^{(n)}, \beta^{(n)}\right)(n=1, \square, \bar{n})$ are generated. Among generated samples, the first $\underline{n}$ samples will be removed. A new set of samples is then defined as a replacement, with its subscriptions as $M=\{\underline{n}+1, \square, \bar{n})$. By applying this approach, joint probability distribution functions $G(\alpha)$ and $G(\beta)$ can be defined:

$$
\begin{aligned}
& G(\alpha)=\frac{\#\left(\alpha^{(n)} \leq \alpha, n \in M\right)}{\bar{n}-\underline{n}}, \\
& G(\beta)=\frac{\#\left(\beta^{(n)} \leq \beta, n \in M\right)}{\bar{n}-\underline{n}},
\end{aligned}
$$


where $\#\left(\beta^{(n)} \leq \beta, n \in M\right)$ is regarded as total number of samples, from which logical expression $\beta^{(n)} \leq \beta, n \in M$ is satisfied. Moreover, expected values of standard variance $\bigsqcup_{i}\left(\beta_{i}\right)$ and covariance matrix $E_{i}\left(\beta_{i}\right)$ are estimated (refer to the formulation in Geweke, 1996):

Confidence intervals of parameter $\alpha$ and $\beta$ are examined and determined by using samples generated from Gibbs sampling. For example, $100(1-2 \varepsilon) \%$ confidence interval of parameter $\alpha$ is defined by using statistical sampling order $\left(\underline{\beta}_{i, m}^{\varepsilon}, \bar{\beta}_{i, m}^{\varepsilon}\right)(i=1, \square, I-1, \quad m=1, \square, M)$ with $\underline{\beta}_{i, m}^{\varepsilon}<\beta_{i, m}<\bar{\beta}_{i, m}^{\varepsilon}$ :

$$
\begin{aligned}
& \underline{\beta}_{i, m}^{\varepsilon}=\underset{\beta_{i, m}^{*}}{\arg \max _{i, m}}\left\{\frac{\#\left(\beta_{i, m}^{(n)} \leq \beta_{i, m}^{*}, n \in M\right)}{\bar{n}-\underline{n}} \leq \varepsilon\right\}, \\
& \bar{\beta}_{i, m}^{\varepsilon}=\underset{\beta_{i, m}^{* *}}{\arg \min _{n}}\left\{\frac{\#\left(\beta_{i, m}^{(n)} \geq \beta_{i, m}^{* *}, n \in M\right)}{\bar{n}-\underline{n}} \leq \varepsilon\right\} .
\end{aligned}
$$

It is noted that the initial value of parameter $\theta^{(0)}$ guarantees for true condition states neither for prior distribution nor for posterior distribution in MCMC simulation. Thus, it is necessary to consider $\bar{n}$ samples generated by Gibbs sampling as posterior distribution of the first $\underline{n}$ set $\theta^{(n)}=\left(\alpha^{(n)}, \beta^{(n)}\right)(n=1, \square, \underline{n})$. When the number of samples increases to $\underline{n}+1$, a hypothetical test using the Geweke statistical test is performed to verify whether samples emanate from the prior or posterior distribution. In the next step, sampling distribution $\theta^{(n)}(n=1, \square, \bar{n})$ is divided into two subsets, $n_{1}$ and $n_{2}$. In the Geweke statistical test, ranges for the two subsets are recommended as $n_{1}=0.1(\bar{n}-\underline{n})$ and $n_{2}=0.5(\bar{n}-\underline{n})$, respectively. According to Chib (1995) and Newey and West (1987), the Geweke statistical test (referred as Z-score) used to verify values of parameter $\alpha$ can be outlined as follows:

$$
\begin{aligned}
Z_{\alpha_{m}^{i}} & =\frac{\bar{\alpha}_{m}^{i}-{ }_{2} \bar{\alpha}_{m}^{i}}{\sqrt{v_{1}^{2}\left(\alpha_{m}^{i}\right)+v_{2}^{2}\left(\alpha_{m}^{i}\right)}}: N(0,1), \\
{ }_{1} \bar{\alpha}_{m}^{i} & =\frac{\sum_{k=\underline{n}+1}^{n+n_{1}} \alpha_{m}^{i, k}}{n_{1}}, \quad{ }_{2} \bar{\alpha}_{m}^{i}=\frac{\sum_{k=n-n_{2}+1}^{\bar{n}} \alpha_{m}^{i, k}}{n_{2}}, \quad v_{1}^{2}\left(\alpha_{m}^{i}\right)=\frac{2 \pi \hat{f}_{\alpha_{m}^{i}}^{1}(0)}{n_{1}}, \quad v_{2}^{2}\left(\alpha_{m}^{i}\right)=\frac{2 \pi \hat{f}_{\alpha_{m}^{i}}^{2}(0)}{n_{2}},
\end{aligned}
$$

where $f_{\alpha_{m}^{i}}^{l}(x)(l=1,2)$ is the probability density function and the value of $2 \pi f_{\alpha_{m}^{i}}^{l}(0)$ is estimated from the following equations:

$$
\begin{aligned}
& 2 \pi \hat{f}_{\alpha_{m}^{i}}^{l}(0)={ }_{1} \hat{\omega}_{0}+2 \sum_{s=1}^{q} w(s, q)_{1} \hat{\omega}_{m}^{i}, \\
& \hat{\omega}_{0}=n_{1}^{-1} \sum_{g=\underline{n}+1}^{\underline{n}+n_{1}}\left(\alpha_{m}^{i, g}-{ }_{1} \bar{\alpha}_{m}^{i}\right)^{2}, \quad{ }_{2} \hat{\omega}_{0}=n_{2}^{-1} \sum_{g=n-n_{2}+1}^{\bar{n}}\left(\alpha_{m}^{i, g}-{ }_{2} \bar{\alpha}_{m}^{i}\right)^{2}, \\
& { }_{1} \hat{\omega}_{m}^{i}=n_{1}^{-1} \sum_{g=\underline{n}+s+1}^{\underline{n}+n_{1}}\left(\alpha_{m}^{i, g}-{ }_{1} \bar{\alpha}_{m}^{i}\right)\left(\alpha_{m}^{i,(g-s)}-{ }_{1} \bar{\alpha}_{m}^{i}\right), \quad{ }_{2} \hat{\omega}_{m}^{i}=n_{2}^{-1} \sum_{g=n-n_{2}+s+1}^{\bar{n}}\left(\alpha_{m}^{i, g}-{ }_{2} \bar{\alpha}_{m}^{i}\right)\left(\alpha_{m}^{i,(g-s)}-{ }_{2} \bar{\alpha}_{m}^{i}\right), \\
& w(s, q)=1-\frac{s}{q+1} .
\end{aligned}
$$


The value of coefficient $q$, which represents the approximate value of spectrum density, should equal 20 , as recommended in the practice of the Geweke statistical test (Geweke, 1996).

In this test, null hypothesis $H_{0}$ and alternative hypothesis $\alpha_{m}^{i}$ concerning the invariance distribution of settingvalues for parameter $\alpha_{m}^{i}$ can be defined as

$$
\left\{\begin{array}{l}
H_{0}:\left|Z_{\alpha_{m}^{i}}\right| \leq Z_{\psi / 2} \\
H_{1}:\left|Z_{\alpha_{m}^{i}}\right|>Z_{\psi / 2}
\end{array}\right.
$$

where $z_{\psi / 2}$ is the critical value to be applied for rejecting the null hypothesis. If the given hypothesis is accepted, the null hypothesis can be defined by a significant level $\psi \%$, to which the condition $z_{\psi / 2} \psi / 2 \%=1-\Phi\left(z_{\psi / 2}\right)$ is satisfied. $\Phi(z)$ is the distribution function of the standard normal distribution. As for hypothetical testing for the distribution of the parameter $\beta_{i, m}(i, m=1, \square, M)$, a similar approach can be applied.

\section{Empirical application}

\subsection{Summary of database}

We applied the hidden Markov model proposed in this study to the surface inspection results of the past 9 years of a general national road managed by $\mathrm{M}$ prefecture, and attempted to predict road rutting deterioration. The targeted database was comprised of data regarding maintenance history and data regarding surface inspection. Surface inspection was conducted every 3 or 6 years. The road area targeted for analysis is approximately $60 \mathrm{~km}$ of a general national road in M prefecture, and measurement samples of $100 \mathrm{~m}$ units were accumulated. From 1986 to the present, rutting measurement has been periodically conducted with measurement vehicles. The managed database used as the applied case of this study is the road surface inspection data of the period between 1998 and 2005. From the data, we focus on the rutting process of the asphalt road. Furthermore, records regarding road maintenance conducted during the period of analysis are also accumulated. For the surface areas where road maintenance was performed, we defined a sample timeline with the beginning point at the initial time, and created a database for the hidden Markov model estimation. Therefore, measurement samples are composed of rutting measurements at the initial time and the following consecutive measurement times, and information regarding the measurement intervals. The database also includes traffic information and road conditions from each measurement sample. A total of 5,261 measurement samples were created in this way.

In general, inspection results of public works are expressed as discrete condition indications, after structural and functional judgment. Therefore, it is possible to express the deterioration process with a Markov chain model. However, the rutting measurement data of the road used in this study are recorded as continuous data. In order to estimate the deterioration prediction model proposed in this study, it is necessary to replace the continuous value of rutting amount with a discrete condition value. In road management, the continuous measurement values of rutting, cracks and irregularity are often changed into several discrete indicators after technical judgment is made. With this study, we categorized the condition of rutting into 5 ratings, shown in Table 1, according to M prefecture's technical policy. Of course, creating discrete categories will cause the deterioration estimation model results to differ. However, from the perspective of road management, it is important to estimate the time it takes to reach the limit of rutting $(15 \mathrm{~mm}$ or $20 \mathrm{~mm})$. We used a rating method different from that shown in Table 1 , and performed a sensitivity analysis regarding the time it takes to reach the rutting limit. As a result, there was no remarkable difference in the prediction results with regard to time, so it was decided to use the condition rating as shown in Table 1.

We refer to the measurement condition values of two continuous times as prior condition and posterior condition. In Table 2 we arranged the measurement samples used in a multi-stage exponential Markov model, with a focus on prior condition and posterior condition. Each row of the table corresponds with prior condition $i$ and each column with posterior condition $j$. Here, we extract samples with no road maintenance between the two measurement times. However, as shown in the table, there are many samples in which the posterior condition exceeds the prior condition. 
In particular, there are many samples in which the prior condition is 2 but the posterior condition is 1 . The fact that road maintenance has not been performed on these samples between the two times is evidence that there are many selection biases in the data for analysis.

\section{\{Table 1 is about here\}}

\{Table 2 is about here

In our empirical study, two objectives are discussed. The first objective is to compare the estimation results of two models (the multi-stage exponential Markov model of Tsuda et al, 2006b and the proposed hidden Markove model) using the same pool of data. The second objective is discussed in section 5.3, where we further compare the estimation results of hidden Markov model on two different cases. In such two cases, we create datasets with assumption of having selection bias and estimate the model's parameters in order to understand the deterioration performance curves in two different cases. From which, we can bencmark for the better model under the situation of having selection bias.

\subsection{Estimation results}

In the empirical study, annual traffic volume of large-sized cars is considered as a main characteristic variable affecting the deterioration or the hazard rate in Eq. (7). Denotation for traffic volume is $x_{i 2}$, which is observable. The first characteristic variable $x_{i 1}$ equals 1 as a constant value representing the overall influence of other variables.

Table 3 presents comparative estimation results of our empirical study using both the multi-stage exponential Markov model (Tsuda et al., 2006b) and the proposed hidden Markov model. It is noted that with multi-stage exponential Markov model, data located above the $45^{\circ}$ line is taken as input. Meanwhile, with the proposed hidden Markov model, all data in Table 2 is considered. Table 3 also highlights the fact that traffic volume exerts a strong influence on deterioration, especially for the first two condition states. The obtained values of parameters with the hidden Markov model are satisfied with credible intervals and convergent criteria in the sense of samples' autogeneration in Bayesian estimation and MCMC simulation. (Values of the Geweke test are less than 1.96.) In the Geweke statistical test, we estimate the z-score values (Geweke, 1996) based on 7000 sampling values of model's parameters (Total number of samples are 10,000, the first 3000 samples are excluded as for burn-in period in order to achieve convergence in the sampling process).

\{Table 3 is about here

\{Table 4 is about here\}

\{Table 5 is about here\}

Further comparing the differences between results of the two models, we estimate the life expectancy of condition states and present the results in Table 4. Relatively, either with the multi-stage exponential Markov model or the hidden Markov model, it can be stated that the hazard rate of condition state 1 is very high. Its high value results in a considerably high speed of deterioration. After about 3 years in operation, road sections with condition state 1 tend to disappear. Condition state 2 has the longest life expectancy, at approximately 12 years. Meanwhile, life expectancies of condition states 3 and 4 are about 8 years. For a better understanding of transition probabilities, we demonstrate the Markov transition probability matrices in Table 5.

Fig. 3(a) presents a comparative picture of deterioration curves. In the figure, the dotted curve represents the decrease in survival probability of corresponding condition states with the multi-stage exponential Markov model. The solid curve is of the estimation results of the hidden Markov model. In view of selection biases, the dotted curve contains bias and does not truly reflect the deterioration progress of the rut index on the entirety of road sections. Its estimation is based purely on the observed data, with embedded selection biases. The solid curve in the figure reflects true deterioration progress, since the hazard rate and life expectancy of condition states are calculated with simulated data. 
Differences in estimation results of the two models are proved from examining displayed outcomes of Table 3 and Fig. 3 (a). As a matter of course, the prediction of deterioration speed in the case of the hidden Markov model has been improved. The deterioration speed is slower than the deterioration speed predicted by the multi-stage exponential Markov model. The distance between two deterioration curves increases along with the operation time of roads. This is due to selection biases that the multi-stage exponential Markov model cannot capture (that is, road inspectors/engineers tend to select worst visual condition states as representative condition states for the entirety of road sections). As a result, a faster deterioration speed occurs in the estimation of the multi-stage exponential Markov model.

Another interesting finding can be drawn from observing deterioration curves in Fig. 3 (a). It is noted that selection biases occur with all condition states. However, the intensity of selection biases increases with higher condition states such as states 3 and 4 . The increasing of selection biases with higher condition states can be viewed as over-estimation of the hazard rates of those states. This problem is likely to happen with road sections which received M\&R activities in the past but for which M\&R records are missing. This finding suggests the necessity for a future study to develop a methodology to capture accurately the transition pattern of performance indexes (road distress) like the rut index.

\{Fig. 3 is about here

\subsection{Estimation bias}

The aim of this section is to compare the estimation results of using the proposed hidden Markov model on two datasets with assumption of having selection bias. With data such as that shown in Fig. 1, the areas below the 45degree line (where the posterior condition measurement improved compared to the prior condition measurement) are suspected to have selection biases. We made 1) a database without the data below the 45-degree line (Truncated $\mathrm{DB})$, and 2) a database with all posterior condition measurements below the 45-degree line matched to the prior condition (so that the posterior condition has not changed from the prior condition) (Censored DB). The results of the estimation are shown in Table 6.

Moreover, Table 7 shows the average hazard rate of the entire target area, using the Truncated DB and Censored DB. By comparing the average hazard rate $E\left[\theta_{i l}\right]$ shown in Table 4 and the average hazard rate of Table 7 , it is possible to see that with both the Truncated DB and Censored DB, the average hazard rate of condition 1 is smaller than the average hazard rate $E\left[\theta_{i l}\right]$ sought with a hidden Markov model. On the other hand, when deterioration has progressed, as in conditions 3 and 4, the average hazard rates of the Truncated DB and Censored DB are larger than with a hidden Markov model, and the deterioration speed is faster. Fig. 3(b) shows performance curves made with the average hazard rates. As can be analogized from the comparison of the average hazard rates, using either the Truncated DB or Censored DB comes with the risk of a deterioration speed too slow with condition 1, and too fast with conditions 3 and 4, compared to the estimation of a hidden Markov model. This shows that with samples in which posterior condition improves compared to prior condition, it is highly possible that the deterioration has not progressed much from the true prior condition measurement to the true posterior condition measurement. Therefore, when using the Truncated DB, samples in which deterioration between the two measurement times progressed slowly might have been deleted from the database. On the other hand, with the Censored DB, because the posterior condition has been matched with the prior condition, (artificial) data with the assumption that deterioration has not progressed have increased; thus, if the prior condition is good, we might assess the deterioration speed as too slow. However, at least with the applied case, regarding the performance curves up to condition 3 , there is no great difference between the performance curves estimated with the Truncated DB and Censored DB, and the performance curve created with the consideration of selection biases.

Regarding the stage where deterioration has progressed, the estimation of deterioration speed is too fast. However, at the stage of progressed deterioration, bias from sample shortage due to road paving maintenance occurs, and there might be a problem with the accuracy of the estimation of the performance curve. With the practical application of road deterioration prediction, the existence of selection biases in the road condition inspection results has already been pointed out. With deterioration prediction, a Truncated DB is created without the data in which the condition improved between two measurement times, and a performance curve is created from this. The applied case in this 
report shows that there is no great practical obstacle in using a truncated DB to estimate a multi-stage exponential Markov model and performance curve, if the condition is at value 3 and rutting is relatively mild. However, this is true for the applied case in this study, and it is necessary to make further efforts to increase experiential knowledge regarding the influence of selection biases on road deterioration prediction.

\{Table 6 is about here

\{Table 7 is about here

\section{Conclusion}

In this paper, we have proposed an innovative analytical methodology to forecast the deterioration process of infrastructure through a hidden Markov model. In the model, selection biases are considered as random variables. Selection biases are eliminated through the assumption of prior and posterior distribution in Bayesian estimation. Furthermore, Markov Chain Monte Carlo simulation is introduced to generate a random sampling population in Bayesian estimation algorithm.

We have presented an empirical study on the Japanese national road system. Estimation results reveal the fact that selection biases have existed in the monitoring data, particularly concerning condition states 3 and 4 .

We further point out the influence of selection bias on deterioration forecasting by comparing the estimation results of multi-stage exponential Markov model of Tsuda et al, 2006b and the proposed hidden Markov model.

However, we have not discussed several points which may serve as topics for extending this study in the future:

- While the present empirical study is carried out on the road system, this model can be applied to various types of infrastructure. Depending on the structural characteristics and prior knowledge of each infrastructure system, selection biases can be considered not as a random variable but also as in the form of a linear function.

- The model can be extended to address the unobservable heterogeneity factor if the hazard rate is considered in the form of a mixture model. The mixture model can be useful in taclking the effects of various factors on measurement errors and selection biases.

- The specification of the probability mass function $f_{i}(\cdot)$ in Eq. (20) can be extended to cover two tails distribution. In another words, we can assume selection bias to distribute on both sides of observable values. However, in order to implement such assumption, we need to come up with analytical solution for the matter called "identification problem", which has been pointed out in econometric research (Kumbhakar and Lovel, 2000)

- Since the estimation results reveal a high risk of having selection biases with condition states 3 and 4 in the monitoring and inspection of road systems, it is suggested that future research should focus on finding the reasons causing selection biases in these condition states.

\section{References}

Ben-Akiva, M., Humplick, F., Madanat, S., Ramaswamy, R., 1993. Infrastructure management under uncertainty: Latent performance approach. Journal of Transportation Engineering 119 (1), 43-58.

Ben-Akiva, M., Ramaswamy, R., May 1993. An approach for predicting latent infrastructure facility deterioration. Transportation Science 27 (2), 174-193.

Capper, O., Moulines, E., Ryden, T., 2005. Inference in hidden Markov models. Springer.

Chib, S., 1995. Marginal likelihood from Gibbs output. Journal of the American Statistical Association 90, 1313-1321.

Chu, C., Durango-Cohen, P., 2007. Estimation of infrastructure performance models using state-space specifications of time series models. Transportation Research Part C 15(1), 17-32.

Chu, C., Durango-Cohen, P., 2008. Estimation of dynamic performance models for transportation infrastructure using panel data. Transportation Research Part B 42(1), 57-81. 
Cochoran, W., Cox, G., 1968. Errors of measurement in statistics. Technometrics 10 (4), 637-666.

Dempster, A., Laird, N. M., Rubin, D. B., 1977. Maximum likelihood from incomplete data via the em algorithm. Journal of the Royal Statistical Society Series B-Vol.39, 1-38.

Diebold, F., Inoue, A., 2001. Long memory and regime switching. Journal of Econometrics 105, 131-159.

Diebolt, J., Robert, C., 1994. Estimation of finite mixture distributions through Bayesian sampling. Journal of the Royal Statistical Society Series B-Vol.56, 363-375.

Durango-Cohen, P., 2007. A time series analysis framework for transportation infrastructure management. Transportation Research Part B 41(1), 493-505.

Geman, S., Geman, D., 1984. Stochastic relaxation, Gibbs distributions and the Bayesian restoration of images. Transactions on Pattern Analysis and Machine Intelligence 6, 721-741.

Geweke, J., 1996. Evaluating the accuracy of sampling-based approaches to the calculation of posterior moments, in: Bernardo, j.m., berger, j.m., dawid, a.p., and smith, a.f.m. (eds.):Bayesian statistics 4. Oxford University Press, 169-193.

Gilks, W., Wild, P., 1992. Adaptive rejection sampling for Gibbs sampling. Applied Statistics 41, 337-348.

Golabi, K., Shepard, R., Febuary 1997. Pontis: A system for maintenance optimization and improvement of us bridge networks. Interfaces 27 (1), 71-88.

Grubbs, F., 1973. Errors of measurement; precision, accuracy, and the statistical comparison of measuring instruments. Technometrics 15 (1), 53-66.

Hamilton, J., 1989. A new approach to the economic analysis of nonstationary series and the business cycle. Econometrica 57 (2), $357-384$.

Hamilton, J., Raul, S., 1994. Autoregressive conditional heteroskedasticity and changes in regime. Journal of Econometrics 64, $307-333$.

Hong, F., Prozzi, J., June 2006. Estimation of pavement performance deterioration using Bayesian approach. Journal of Infrastructure System 12 (2), 77-86.

Humplick, F., April 1992. Highway pavement distress evaluation modeling measurement error. Transportation Research Part B 26 (2), $135-154$.

Ibrahim, J., Ming-Hui, C., Sinha, D., 2001. Bayesian Survival Analysis. Springer Series in Statics.

Jeff, G., 2006. Bayesian Methods: A Social and Behavioral Sciences Approach, 2nd Edition. Chapman and Hall/CRC Taylor and Francis Group.

Kalman, R.E., 1960. A New Approach to Linear Filtering and Prediction Problems. Transaction of the ASME-Journal of Basic Engineering, 82(D), 35-45.

Kim, C.-J., Nelson, C., 1999. State-space models with regime switching : Classical and Gibbs-sampling approaches with applications. MIT Press.

Kumbhakar, S. C., Lovell, C., 2000. Stochastic Frontier Analysis. Cambridge University Press.

Lancaster, T., 1990. The econometric analysis of transition data. Cambridge University Press.

Lawrence, R., February 1989. A tutorial on hidden Markov models and selected applications in speech recognition. Proceedings of the IEEE 77 (2).

MacDonald, I., Zucchini, W., 1997. Hidden Markov and Other Models for Discrete-valued Time Series. Chapman and Hall.

Madanat, S., Ben-Akiva, M., 1994. Optimal Inspection and Repair Policies for Infrastructure Facilities. Transportation Science 28 (1), 55-62.

Madanat, S., Mishalani, R., Ibrahim, W., 1995. Estimation of infrastructure transition probabilities from condition rating data. Journal of Infrastructure Systems 1 (2), 120-125.

Mishalani, R., Gong, L., March 2009. Optimal infrastructure condition sampling over space and time for maintenance decision-making under uncertainty. Transportation Research Part B 43 (3), 311-324.

Newey, W. K., West, K. D., 1987. A simple, positive semi-definite, heteroskedasticity and autocorrelation coisistent covariance matrix. Econometrica 55, 703-708.

Robert, C., 1996. Mixtures of Distributions: Inference and Estimation, in: Gillks, W.R., Richardson, S. and Spiegelhalter,

Robert, C., Ryden, T., Titterington, D.M., 2000. Bayesian inference in hidden Markov models through the reversible jump Markov chain monte carlo method. Journal of the Royal Statistical Society Series B-Vol.62, 57-75.

Scott, S. L., 2000. Bayesian methods for hidden Markov models: Recursive computing in the 21st century. Journal of the American Statistical Association 97, 337-351.

Shahin, M., 2005. Pavement Management for Airports, Roads, and Parking Lots. Springer.

Shin, H. C., Madanat, S. M., 2003. Development of stochastic model of pavement distress initiation. Journal of Infrastructure Planning and Management (744/IV-61), 61-67.

Titterington, D., Smith, A., Makov, U., 1985. Statistical Analysis of Finite Mixture Distributions. John Wiley and Sons.

Tsuda, T., Kaito, K., Yamamoto, H., Kobayashi, K., 2006a. Bayesian estimation for Weibull deterioration hazard model. Journal of Civil Engineering, JSCE (in Japanese) (No.798/VI-68), 125-136.

Tsuda, Y., Kaito, K., Aoki, K., Kobayashi, K., 2006b. Estimating Markovian transition probabilities for bridge deterioration forecasting. Journal of Structural Engineering and Earthquake Engineering 23 (2), 241-256. 
Zucchini, W., MacDonald, L. L., 2009. Hidden Markov Models for Time Series. Taylor and Francis. 